\title{
On Closure Relations for Dynamic Vapor Compression Cycle Models
}

\author{
Christopher R. Laughman Hongtao Qiao \\ Mitsubishi Electric Research Laboratories \\ Cambridge, MA, USA \\ \{laughman, qiao\}@merl.com
}

\begin{abstract}
Models of closure relations, or the expressions that relate the heat transfer coefficients and frictional pressure losses to other variables of the vapor-compression cycle, can have a significant impact on the performance on the overall cycle behavior. We explore three different approaches that may be used in formulating these closure models, and show that approaches that impose a nonlinear algebraic coupling can impose significant computational challenges. In comparison, models that incorporate lowpass dynamics can effectively decouple this nonlinear behavior, resulting in simulations that are faster and demonstrate more realistic and robust behavior.

Keywords: Modelica, heat pump, vapor compression cycle, approximation
\end{abstract}

\section{Introduction}

At the heart of modeling lies the art of approximation. Models of physical phenomena are always driven by a set of requirements that may relate to an exploration of possible system architectures, designing controls, or a variety of other possible purposes. These requirements drive the formulation of the model, so that the complexity and computational speed of the model must be balanced against the accuracy requirements.

This tradeoff can be readily observed from even a cursory survey of the two dominant methodologies for dynamically modeling heat exchangers: moving boundary models and finite volume models. These methodologies have different levels of complexity and requirements for simulation time: finite volume models describe the heat exchanger behavior accurately at a fine spatial and temporal resolution at a large cost of simulation time, while moving boundary models lump the spatial behavior of heat exchangers into a limited set of up to three fluid zones, and are correspondingly fast. Both of these methodologies are appropriate for different purposes, and the modeling engineer will usually choose the fastest approach with sufficient accuracy according to the specific requirements of the application.

The description of local heat transfer coefficients (HTCs) and frictional pressure losses (commonly referred to as closure relations, due to the fact that they "close" the system of equations so that the number of equations equals the number of variables) in these models can be particularly challenging, as the correlations developed to most accurately describe experimentally observed phenomena are usually formulated with accuracy as the primary concern, and with little regard for computational considerations. Consequently, they can be difficult to incorporate into system-level models of thermofluid systems as they may be extremely nonlinear, tend towards infinity as mass flow rates go to zero, or exhibit other problematic behavior. These correlations are also usually defined only for specific flow conditions or refrigerant phases, so that there will inevitably be significant discontinuities between regions of the validity for specific correlations. Moreover, dynamic simulation presents additional difficulties as the unknown refrigerant mass flow rates, pressures, and specific enthalpies preclude the use of any initial information about the phase of the refrigerant (condensation, evaporation, liquid, or vapor) or the flow regime (laminar or turbulent), so the correlations must be defined in a manner which encompasses a wide range of flow conditions.

A variety of different approaches have previously been proposed to manage these closure relations both for steady-state and dynamic simulation. Perhaps the most straightforward of these approaches involves the creation of simplified correlations with improved behavior that have parameters that are tuned to approximate the original correlations. This method is used in a wide variety of literature (Qiao et al., 2015), and results in simulations that match observed experimental behavior quite well. Cycle models, in particular, require formulations of HTCs or frictional pressure losses that cover wide ranges of flow conditions, and the use of interpolation methods to smoothly stitch together correlations governing specific sets of conditions across transition regions has been used quite successfully (Elmqvist et al., 2003). An alternative approach was also proposed by Laughman et al. (2016) in which a nonparametric kernel regression method was used to approximate heat transfer coefficients directly from data, and the resulting simulations were shown to work well.

Despite the general success of these methods, simulations of vapor compression cycle models that employ these approaches can still exhibit problematic dynamics 


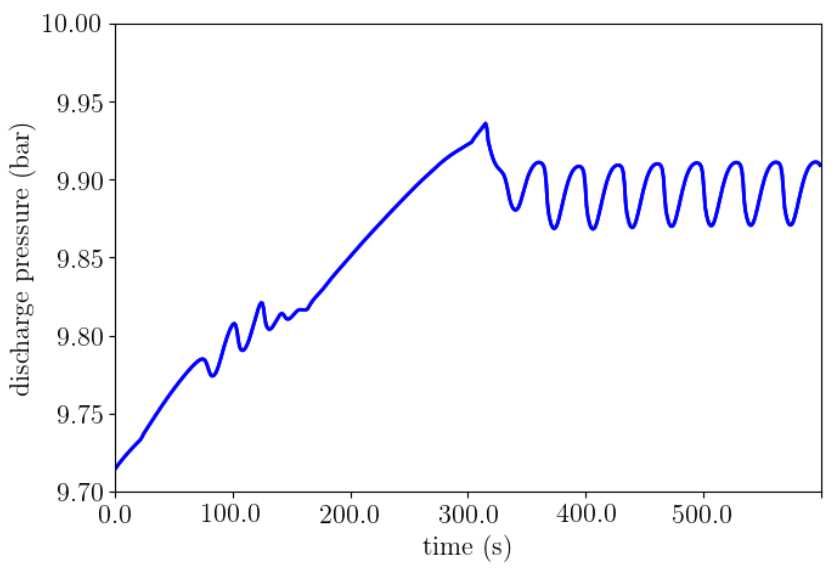

Figure 1. Oscillations in compressor outlet pressure after initialization with constant inputs.

that result in slow execution times and demonstrate nonphysical behavior. One example is provided in Figure 1, which illustrates a periodic oscillation in the compressor outlet pressure that can arise when the system of differential algebraic equations (DAEs) representing the cycle behavior is initialized and driven with constant inputs. These oscillations, which can occur in either the presence or absence of any external forcing function, are related to interactions between a simplified HTC model and the rest of the system dynamics, and will be discussed further in Section 3. This spurious behavior, which is not observed in experiments, will affect many other variables in the cycle and significantly increase simulation times. Because these oscillations can be shown to be related to the closure models used, alternative approaches for describing the heat transfer coefficient and frictional pressure drop may be of interest to the modeling engineer.

In this work, we develop models of the vapor compression cycle in Modelica (Modelica Association, 2017) to investigate the effect that different closure relation models have on the overall system, and demonstrate that the addition of low-pass dynamics to the closure models can significantly improve the performance of the cycle simulations, particularly for the heat transfer coefficient. In Section 2, we develop models of the components used in the vapor compression cycle, and then study the effect of the original closure models on the overall cycle dynamics as well as the modified closure models with the added dynamics in Section 3. Section 4 provides a brief treatment of some results indicating the efficacy of these methods, and then a brief set of concluding remarks is presented in Section 5.

\section{Component \& System Models}

We focus on the simulation of simple vapor compression cycles in this work, such as are used in many contemporary air-conditioning and heat pumping systems; a schematic illustrating a prototype cycle that includes a condensing tube-fin heat exchanger (HEX), an evaporat-

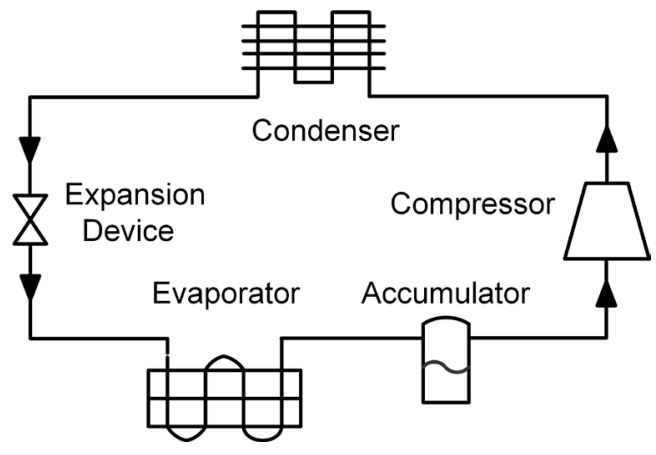

Figure 2. Basic vapor compression cycle.

ing tube-fin HEX, a compressor, and a linear expansion valve (LEV) is illustrated in Figure 2. Because the temporal behavior of the cycle is dominated by the HEXs over the time scales of interest, the system models in this work used dynamic models of the HEXs and static (algebraic) models of the compressor and expansion valves. Finite volume models (Li et al., 2014) were used for the HEXs to capture the dynamic behavior of the refrigerant pressures, as well as the spatially-dependent characteristics of these components. We assume 1-D refrigerant flow so that properties only vary along the length of the pipes; we also assume that the refrigerant can be described as a Newtonian fluid, negligible viscous dissipation and axial heat conduction in the direction of flow, negligible contributions to the energy equation from the kinetic and potential energy of the refrigerant, negligible dynamic pressure waves in the momentum equation, and thermodynamic equilibrium in each two-phase refrigerant volume.

Under these assumptions, the partial differential equations describing the conservation of mass, momentum, and energy (Levy, 1999) for the refrigerant can be spatially discretized for these finite volume models. A staggered grid scheme, illustrated in Figure 3, is used to avoid nonphysical pressure variations caused by numerical artifacts by decoupling the mass and energy equations computed for the volume cells (represented by the black solid boundary) from the momentum equations computed for the flow cells (represented by the red dashed boundary). Integration of these equations across these cells, as well as the use of the upwind difference method to approximate refrigerant properties for the convection-dominated flows from this application, results in a set of ordinary differential equations describing the conservation equations, as given in Equations 1, 2, and 3.

$$
\begin{aligned}
A_{c} \Delta z \rho_{i}= & \dot{M}_{i-1 / 2}-\dot{M}_{i+1 / 2}, \\
\Delta z \frac{\mathrm{d} \dot{M}_{i+1 / 2}}{\mathrm{~d} t}= & \dot{I}_{i}-\dot{I}_{i+1}-A_{c}\left(p_{i+1}-p_{i}\right) \\
& -P \Delta z \bar{\tau}_{w, i+1 / 2}, \\
A_{c} \Delta z u_{i}= & \dot{M}_{i-1 / 2}\left(h_{i-1 / 2}-\bar{h}_{\rho, i}\right) \\
& -\dot{M}_{i+1 / 2}\left(h_{i+1 / 2}-\bar{h}_{\rho, i}\right)+P \Delta z q_{i}^{\prime \prime},
\end{aligned}
$$




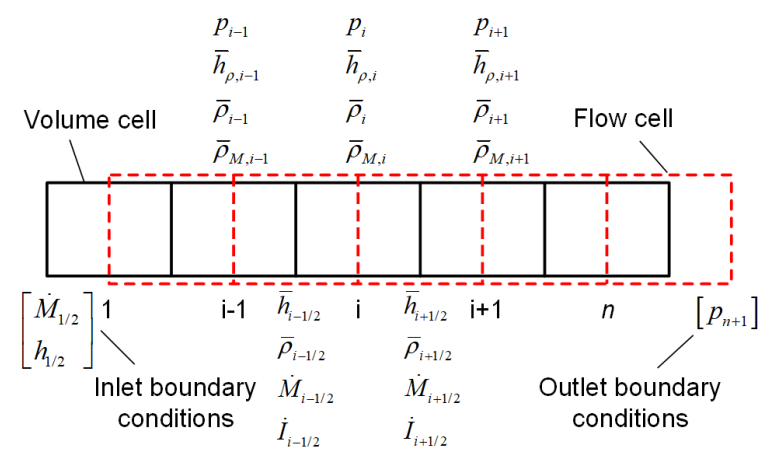

Figure 3. Finite volume discretization of refrigerant pipe.

where $\bar{\rho}_{M}$ represents the momentum density, $\bar{h}_{\rho}$ and $\bar{h}$ signify the the density-weighted and flow-weighted specific enthalpies, the wall shear stress $\bar{\tau}=\frac{1}{2} f \bar{\rho} u|u|$ and $f$ is the Fanning friction factor, and $P$ is the circumference of the flow channel. The closure model for the frictional pressure term $f$ will be provided in Section 3, and symbols with overbars represent average quantities in each cell. The dynamic states used in this model include the refrigerant pressures $p$, density-weighted specific enthalpies $\bar{h}_{\rho}$, and densities $\rho$ (Laughman et al., 2017).

Because the fully dynamic momentum balance adds considerable complexity to the description of the system so that the system description consists of $3 n$ ODEs where there are $n$ volumes per HEX, a number of different approximations to the momentum balance have been be developed to reduce this complexity and speed up these simulations (Qiao and Laughman, 2018). We used three variants in this study, including a friction-only formulation, the assumption of a uniform $d p / d t$, and the assumption of a linear pressure distribution. In the friction-only formulation, we assume that the derivative in Equation 2 is negligible due to the inertia term's minor importance to the thermal behavior of the system, and also neglect the acceleration pressure loss and gravity effect (Brasz and Koenig, 1983), since both of them are typically smaller than the frictional pressure loss. This yields a momentum balance of the form

$$
p_{i+1}=p_{i}-\frac{P}{A_{c}} \Delta z \bar{\tau}_{w, i+1 / 2}
$$

In this formulation, we assume that the number of state variables are still $2 n$ and the mass flow rates are the algebraic variables.

In the uniform $d p / d t$ approach, the fact that the acoustic waves propagate with a speed of sound in the direction of fluid flow is used to motivate the assumption that the time derivatives of pressures are spatially invariant along the direction of flow. As a result, the number of dynamic pressure states is reduced to a single numerical state per pressure level, yielding a very efficient system of equations for each control volume, since $d p / d t$ is given as an input. Note that the time derivative of pressure is not treated as constant over time, but rather in space along the direction of flow. The pressure distribution in the heat exchanger still depends on the selected pressure loss models, and thus on the mass flow rates (Richter, 2008).

Since $\frac{\mathrm{d} p_{1}}{\mathrm{~d} z}=\frac{\mathrm{d} p_{n+1}}{\mathrm{~d} z}=\frac{\mathrm{d} p_{r e f}}{\mathrm{~d} z}$, the equations of continuity and energy become

$$
\begin{aligned}
& A_{c} \Delta z\left(\frac{\partial \bar{\rho}_{i}}{\partial p_{i}} \frac{\mathrm{d} p_{r e f}}{\mathrm{~d} t}+\frac{\partial \bar{\rho}_{i}}{\partial \bar{h}_{\rho, i}} \frac{\mathrm{d} \bar{h}_{\rho, i}}{\mathrm{~d} t}\right)=\dot{M}_{i-1 / 2}-\dot{M}_{i+1 / 2} \\
& A_{c} \Delta z\left(\bar{\rho}_{i} \frac{\mathrm{d} \bar{h}_{\rho, i}}{\mathrm{~d} t}-\frac{\mathrm{d} p_{r e f}}{\mathrm{~d} t}\right)=\dot{M}_{i-1 / 2}\left(h_{i-1 / 2}-\bar{h}_{\rho, i}\right) \\
& -\dot{M}_{i+1 / 2}\left(h_{i+1 / 2}-\bar{h}_{\rho, i}\right)+P \Delta z q_{i}^{\prime \prime}
\end{aligned}
$$

In this approach, only specific enthalpies are the differential variables, and therefore the total number of dynamic states is reduced from $3 n$ by $n-1$.

In comparison, the linear pressure loss approach assumes that the pressure is linearly distributed along the heat exchanger (Jensen, 2003), i.e., $p_{i}=p_{1}+(i-$ 1) $\frac{p_{n+1}-p_{1}}{n}$. Therefore, one can obtain

$$
\frac{\mathrm{d} p_{i}}{\mathrm{~d} t}=\left(1-\frac{i-1}{n}\right) \frac{\mathrm{d} p_{1}}{\mathrm{~d} t}+\frac{i-1}{n} \frac{\mathrm{d} p_{n+1}}{\mathrm{~d} t} .
$$

Aggregating all local momentum balances for flow cells 1 to $n$ results in

$$
\begin{aligned}
n \Delta z \frac{\mathrm{d} \overline{\dot{M}}}{\mathrm{~d} t}= & \dot{I}_{1}-\dot{I}_{n+1}-A_{c}\left(p_{n+1}-p_{1}\right) \\
& -P \Delta z \sum_{i=1}^{n} \bar{\tau}_{w, i+1 / 2}-g A_{c} \Delta z \sum_{i=1}^{n} \bar{\rho}_{i+1 / 2} \sin \theta_{i}
\end{aligned}
$$

where $\overline{\dot{M}}$ is the average mass flow rate $\frac{1}{n} \sum_{i=1}^{n} \dot{M}_{i+1 / 2}$. This variant can be further simplified by summing up the momentum equation with only frictional pressure loss instead for all the flow cells. This variant only has $n+3$ dynamic states.

The refrigerant wall is modeled as one-dimensional heat conduction in the direction perpendicular to the refrigerant flow, with convective boundary conditions described by the refrigerant-side and air-side heat transfer coefficients, which will also be given in Section 3. This wall element can be modeled simply by

$$
\frac{d\left(M_{w} c_{w}\right)}{d t}=\frac{k_{w} A_{s}\left(T_{a}-T_{w}\right)}{L_{w} / 2}+\frac{k_{w} A_{s}\left(T_{b}-T_{w}\right)}{L_{w} / 2},
$$

and the surface wall temperatures $T_{a}$ and $T_{b}$ are related to the bulk temperature of the adjacent fluid by

$$
Q=\alpha A\left(T_{\text {fluid }}-T_{\text {surf }}\right) .
$$

A multicomponent ideal gas moist-air model was used for the air-side of this work. The mass and energy conservation equations used to describe the heat transfer from the outer surface of the tubes to the air reflected this multicomponent model, as described by Equation 12, where the 
mass transfer coefficient was given by a modified Lewis correlation.

$$
\begin{aligned}
\dot{M}_{\text {air }} c_{p, \text { air }} \frac{d T_{\text {air }}}{d y} \Delta y= & \alpha_{\text {air }}\left(A_{o, \text { tube }}+\eta_{\text {fin }} A_{o, f i n}\right)\left(T_{w}-T_{\text {air }}\right) \\
\dot{M}_{\text {air }} \frac{d \omega_{\text {air }}}{d y} \Delta y= & \alpha_{m}\left(A_{o, \text { tube }}+\eta_{\text {fin }} A_{o, f i n}\right) \times \\
& \min \left(0, \omega_{\text {water }, \text { sat }}-\omega_{\text {air }}\right)
\end{aligned}
$$

A simple isenthalpic model of the electronic expansion valve was also used, as described by a standard orifice flow equation

$$
\dot{M}=C_{v} a_{v} \sqrt{\rho_{\text {in }} \Delta P},
$$

where the mass flow rate is regularized in the neighborhood of zero flow to prevent the derivative of the mass flow rate from tending toward infinity. The flow coefficient $C_{v}$ is generally determined via calibration against experimental data, while the flow area $a_{v}$ represents the control authority over the orifice size.

All cycle models in this work included a variable-speed high-side rotary compressor. We used simplified 1-D models of this component to describe the system due to the complex nature of the heat transfer and fluid flow through the compressor. Its performance was described by relating the volumetric efficiency $\eta_{v}$ and isentropic efficiency $\eta_{i s}$ to the suction pressure $P_{s u c}$, discharge pressure $P_{d i s}$, and compressor frequency $f$, as given by

$$
\begin{aligned}
\eta_{v} & =\frac{\dot{M}_{\text {comp }}}{\rho_{\text {suc }} V f} \\
\eta_{\text {is }} & =\frac{h_{\text {dis }, i s e n}-h_{\text {suc }}}{h_{\text {dis }}-h_{\text {suc }}} .
\end{aligned}
$$

The compressor power consumption $\dot{W}$ was also related to the compressor speed and the ratio of inlet and outlet pressures, i.e., $\dot{W}\left(P_{r a t}, \omega\right)$. The coefficients used for the functional forms of $\eta_{v}, \eta_{i s}$, and $\dot{W}$ were derived from experimental data, and the expressions themselves are provided in (Laughman et al., 2017).

Standard fan laws (ASHRAE, 2008) were used to describe the behavior of the heat exchanger fans, in which the volumetric flow rate was assumed to be directly proportional to the fan speed, while the power consumed by the fan was assumed to be proportional to the cube of the fan speed. These simple algebraic models were scaled by experimentally measured values of fan speed, flow rate, and power for a representative system; to minimize the error in these fits, linear and quadratic terms were also included in the power model to account for observed variations in the data.

A simple room model was also used to study the dynamics of the different cycle models on the idealized model of an occupied space. A lumped model of the room air was used to describe the sensible and latent dynamics of the space, and the room model was coupled to the

\begin{tabular}{lc} 
Parameter & Value \\
\hline Refrigerant & R134A \\
Total refrigerant mass $(\mathrm{kg})$ & 1.65 \\
condensing HEX tube diameter $(\mathrm{mm})$ & 7.9 \\
evaporating HEX tube diameter $(\mathrm{mm})$ & 6.3 \\
condensing HEX tube length $(\mathrm{m})$ & 0.5 \\
evaporating HEX tube length $(\mathrm{m})$ & 2.5 \\
condensing HEX number of tubes & 27 \\
evaporating HEX number of tubes & 10
\end{tabular}

Table 1. Geometric parameters of the vapor compression cycle under consideration.

ambient environment through a simple RC circuit model of a building envelope with convective heat transfer on both the outside and inside interfaces of the envelope. Selected information about some of the important geometric parameters of the system is provided in Table 1.

\section{Closure Models}

In the previous section, we described the componentbased models of the conservation equations that describe the behavior of all of the components: the heat exchangers, the expansion valve, and the compressor. As suggested in the introduction, however, the closure models used to relate the heat transfer coefficient and the frictional pressure loss to other variables in the cycle can play an important role in the overall cycle dynamics. We therefore study three types of closure models in this section: full algebraic models that are developed directly from correlations published in the literature, simplified algebraic models that are related to the previous correlation-based models but have a much simpler mathematical form, and dynamic models that also include time-dependent effects. These methods will each be described in turn, and then the results of implementing each of them in a complete vapor-compression cycle will be discussed in Section 4.

\subsection{Full algebraic models}

The most straightforward approach to describing closure models involves the direct implementation of the original HTC and frictional pressure loss correlations from the literature. Because these variables are dominated by microscopic phenomena, first principles-based models are generally either too analytically difficult to formulate or too computationally difficult to simulate, so there is a strong tradition of measuring these phenomena experimentally and fitting mathematical formulas to the resulting data. Because the parametric dependence of these relations changes depending on the nature of the flow and the refrigerant, these correlations are usually formulated for specific flow conditions, such as single-phase, two-phase, laminar, and/or turbulent conditions, and are only valid for that type of flow. We used a variety of these correlations to describe the flow, as described in Table 2. 


\begin{tabular}{ll} 
Correlation Type & Correlation \\
\hline $1 \phi$ liquid HTC & Dittus and Boelter (1930) \\
Condensing HTC & Dobson and Chato (1998) \\
Boiling HTC & Gungor and Winterton (1987) \\
$1 \phi$ vapor HTC & Dittus and Boelter (1930) \\
$1 \phi$ laminar $\Delta P_{f}$ & Blasius (Stephan, 2010) \\
$1 \phi$ turbulent $\Delta P_{f}$ & Hagen-Poiseuille (Stephan, 2010) \\
Condensing $\Delta P_{f}$ & Lockhart and Martinelli (1949) \\
Boiling $\Delta P_{f}$ & Jung and Radermacher (1989)
\end{tabular}

Table 2. Correlations used to describe heat transfer coefficients and frictional pressure losses.

The nature of dynamic simulation makes it very difficult to ensure that a specific set of conditions will always be met, due to the challenges of initializing large systems of DAEs and the fact that the flow conditions may change dynamically so that a given cell in the finite volume HEX model sometimes experiences single-phase conditions and sometimes experiences two-phase conditions. In addition, it is computationally beneficial if the correlations are at least $C^{1}$ continuous over a wide range of operation, to allow the integrators to function efficiently. Discontinuities in the closure models may also require the integrator to do event iteration or generate nonlinear sets of equations to solve.

We therefore blend these correlations together via a univariate trigonometric interpolation method (Richter, 2008). In this method, the user defines transition zones between regions of validity for the individual correlations according to some given variable that will be used to determine which correlation to use. The output of the method is a $C^{2}$ continuous function that smoothly transitions between different correlations, depending on the value of the transition variable. For example, the condensing and boiling heat transfer coefficients are combined into a unified two-phase HTC that uses the value of the temperature difference between the refrigerant and the pipe wall to smoothly transition between these two flow regimes. If $\Delta T$ is greater than $T_{c}$ degrees $\mathrm{C}$, then the condensing heat transfer coefficient is used, while if $\Delta T$ is less than $T_{b}$ degrees $\mathrm{C}$, the boiling heat transfer coefficient is used. These values are smoothly interpolated in a transition region from $T_{b}<0<T_{c}$.

This unified two-phase HTC is then itself blended with the liquid and vapor heat transfer coefficients by using the flow quality $x$ as the transition variable and transition regions on either side of the two-phase region. If $x<0$ the liquid heat transfer coefficient is used, while if $0<x<x_{1}$ then there is a smooth transition from the liquid heat transfer coefficient to the two-phase heat transfer coefficient. Similarly, if $x_{2}<x<1$ there is a smooth transition between the two-phase heat transfer coefficient and the vapor heat transfer coefficient, and the vapor heat transfer coefficient is used if $x>1$. The resulting blended heat transfer coefficient then is able to accurately describe the

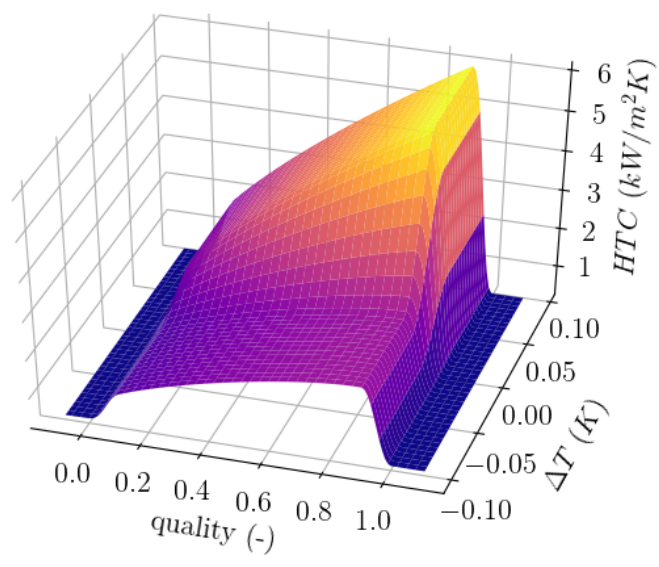

Figure 4. Blended HTC as a function of the wall temperature difference $\Delta T$ and the flow quality $x$.

flow over all flow regimes. A similar approach is also used to unify the different frictional pressure loss correlations into a blended correlation covering a wide range of flow regimes.

The efficacy of this approach can be seen in Figure 4, which illustrates the HTC as a function of the temperature difference $\Delta T$ between the refrigerant and the tube wall as well as the flow quality $x$ of the refrigerant. This surface was mapped out by using the correlations provided in Table 2; the temperature transition region between boiling and condensation was set to $-0.025 \mathrm{~K} \leq \Delta T \leq 0.025 \mathrm{~K}$, and the transition regions between both single-phase regions and the two-phase region were set to $0.05 \mathrm{~kg} / \mathrm{kg}$. These transitions can be clearly seen by considering slices parallel to the quality axis: the HTC increases at the transition from the subcooled liquid to the two-phase regions, gradually continues to increase as the flow quality increases, and then rapidly decreases at the transition between the two-phase and superheated vapor region. Note the large slope of the transition between the two-phase and vapor regions, which will be discussed in more detail in the following sections.

While these somewhat Frankenstein-like equations might be said to most accurately represent the relation $\alpha_{i}=f(\cdot)$ according to the literature, the algebraic relations between the system inputs and the heat transfer coefficient $\alpha_{i}$ can be extremely nonlinear. When these functions are then compiled into a system model, these sets of equations are often solved using a nonlinear solver such as the multivariate Newton-Raphson method, which can be both timeconsuming and fragile. Moreover, each volume in the heat exchanger will incorporate these nonlinear blocks, adding further complexity to the overall description of the system.

\subsection{Simplified algebraic models}

One alternative approach that has been successfully used to mitigate these nonlinearities has been the creation of simplified algebraic closure models that capture the gen- 
eral trends of the detailed heat transfer coefficient and frictional pressure loss relations without implementing their complexity. A wide variety of forms can be used for these relations, depending on the required parametric dependence or level of fidelity to the behavior of the original correlations. For example, we used a simplified heat transfer relation for each phase according to

$$
\alpha=\alpha_{0}\left(\frac{\dot{M}}{\dot{M}_{0}}\right)^{b}
$$

The constants $\alpha_{0}$ for the liquid, two-phase, and vapor flow regions were calculated by coarsely approximating the behavior of the full correlations over their regions of validity, and the same trigonometric interpolation method was used to smoothly transition between phases.

This method is powerful because it provides a small number of parameters to tune to the original correlations, as well as the widths of the transition regions, but the number of state variables that are coupled in these models is much smaller than is the case with the original correlations. This makes it possible to significantly improve the performance of the simulations by eliminating the large number of blocks of nonlinear equations. It is important to note that these simplified correlations still manifest very large changes in the heat transfer coefficient, due to the fact that the two-phase heat transfer coefficients can be more than an order of magnitude greater than the singlephase heat transfer coefficients. These large changes result in very large magnitudes in $d \alpha / d x$, which can also affect the system simulations.

Similar simplified formulations were also constructed for the frictional pressure loss, which was expressed as

$$
\Delta p_{f}=K \Delta p_{0}\left(\frac{\dot{M}}{\dot{M}_{0}}\right)^{b},
$$

where $b=2$ for these models. The Colebrook correlation for the single-phase friction factor and the Friedel correlation for two-phase multipliers were used to determine the nominal values of $K, \Delta p_{0}$, and $\dot{M}_{0}$. This relation is not only less nonlinear than the original correlation-based relations, but it is also easily invertible and can allow the pressure loss to be calculated as a function of the mass flow rate, or vice versa. As such, the resulting systems simulations had much faster performance, since the nonlinear dependence on the variety of input variables was removed from the relation and the integrator could take much larger steps.

\subsection{Dynamic models}

The main challenges posed by the algebraic approaches used to formulate closure models in the previous section are the nonlinear sets of algebraic equations, which may couple together multiple state variables, and the potential effect of large gradients in the transition regions. The sets of nonlinear equations can present a particular problem for the solvers, as the successful simulation of a model depends on the quality of nonlinear solvers used by a particular Modelica tool, as well as the initial guesses used by that method. This tool-dependent aspect of these models can potentially cause simulations of the same model in different tools to yield different answers, which is rather problematic.

Rather than implement these nonlinear algebraic relations, we propose the incorporation of dynamics into the closure models to decouple the HTC or frictional pressure loss from the other state variables. This will make the closure variables into state variables of the system, and will decouple the value of the closure variable in the fluid computations with the value of the closure variable calculated from the other state variables. In the case of the heat transfer coefficient, this may be calculated by

$$
\begin{aligned}
\hat{\alpha} & =f(\dot{M}, p, h) \\
\frac{d \alpha}{d t} & =\frac{1}{\tau}(\hat{\alpha}-\alpha),
\end{aligned}
$$

where $f$ denotes the algebraic HTC correlation, $\hat{\alpha}$ represents the algebraic HTC, and $\alpha$ represents the lowpassed version of the HTC. Since this heat transfer coefficient is now a state variable, it needs to be initialized to an initial value $\alpha_{0}$ at $t=0$. We expect that this will eliminate many of the nonlinear equation blocks and also reduce the sensitivity of the system to the large gradients, as the changes in the heat transfer coefficient will now also depend on its previous values. The parameter $\tau$ should be tuned to be substantially faster than other time constants of the system in order to ensure that it will not change the system response.

These low-pass dynamics are often reasonable to include because the bandwidth of experimental vapor compression cycles is often much lower than is observed in simulations. Many potential sources of modeling error could potentially contribute to this discrepancy. For example, the working fluid in many practical cycles is a mixture of both refrigerant and oil, which is needed for lubrication of the compressor. The presence of this oil in heat exchangers will damp sudden changes in the refrigerant state, as refrigerant must diffuse into or out of the oil in response to changes in the refrigerant properties. In addition, these systems often have many time delays and couplings, such as axial heat conduction and diffusion, that are often not incorporated into dynamic models but are inherently low-pass in nature. In general, we do not advocate blindly adding dynamics to existing system models because these added dynamics will be convolved with original dynamics, but the tradeoff between inaccuracies of the added dynamics and the inaccuracies of simplified methods, not to mention the improvement in computational speed, may motivate the use of these approximations in some cases.

The implementation in Modelica is exceedingly simple, and is attractive from an object-oriented design perspec- 


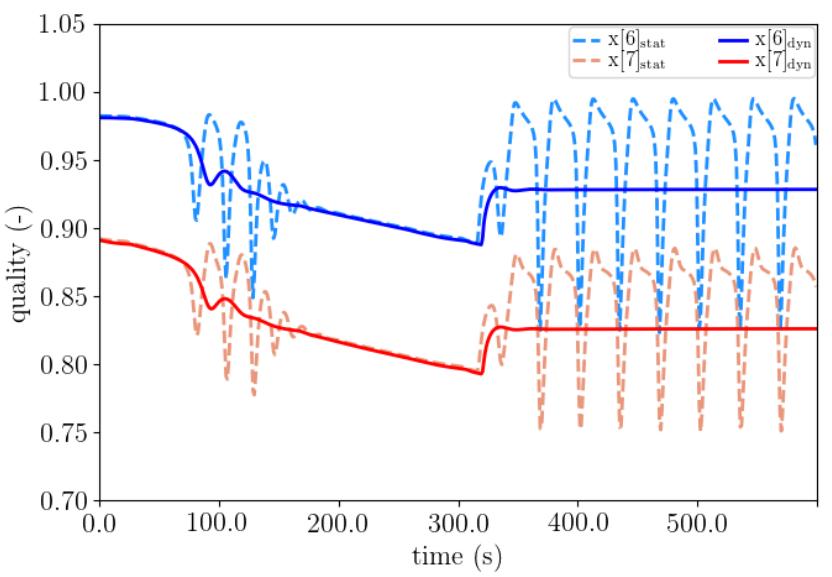

Figure 5. Refrigerant flow quality $x$ in volumes 6 and 7 for simple algebraic HTCs and detailed dynamic HTCs.

tive. The code in the original correlation function that assigns the closure variable,

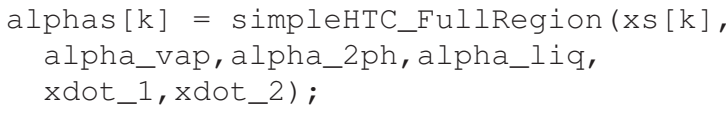

need only be changed to incorporate these dynamics in the output, e.g.,

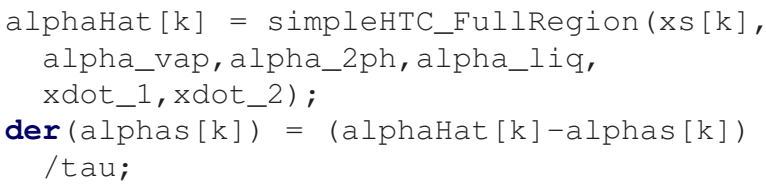

and the additional state equations will be incorporated into the model. This type of modification would be much more difficult to implement in a non equation-oriented language, as it would necessitate the complete rearrangement of the state vector.

\section{Results}

The effect of these different closure relations was studied by implementing the full vapor-compression cycle model in the Modelica language and comparing the resulting cycle behavior when different closure models were incorporated into the complete cycle model. These simulations were performed on a desktop with an Intel i7 processor with $32 \mathrm{~Gb}$ of RAM using the Dymola 2018 FD01 compiler (Dassault Systemes, AB, 2018), and the differential algebraic equations were integrated with the DASSL solver with the tolerance set to $10^{-5}$. All of the models for this application were developed by the authors with the exception of the refrigerant property models, which were obtained from the commercial Vapor Cycle Library (Modelon AB, 2018).

As the cycle behavior with the alternate heat transfer coefficient models differs from the behavior with the alternate frictional pressure drop models, we discuss each of

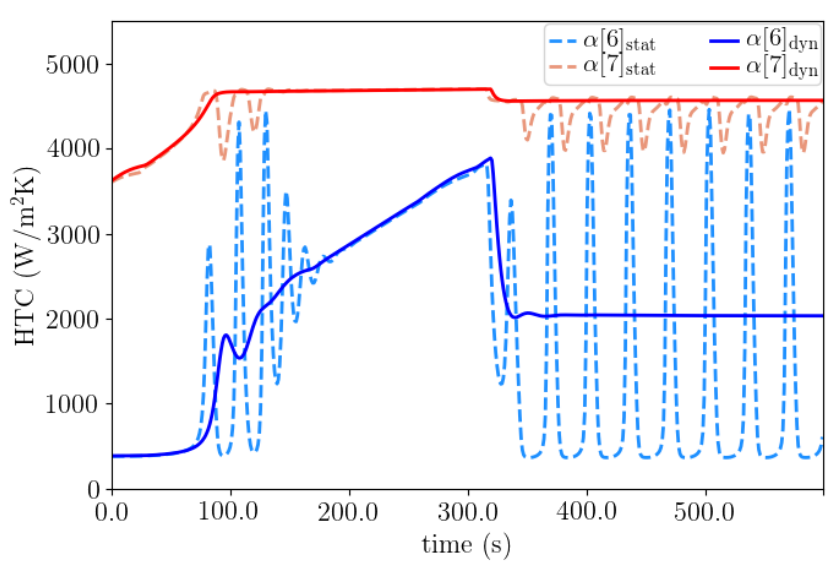

Figure 6. Refrigerant-side heat transfer coefficients $\alpha_{i}$ for volumes 6 and 7 for simple algebraic HTCs and detailed dynamic HTCs.

these types of closure models in their own respective subsections. While the base cycle model was identical in all of these experiments, the friction-only momentum balance was implemented with the simplified algebraic pressure drop model when heat transfer coefficient models were studied, while the simplified dynamic heat transfer coefficient model was used when the different pressure drop models were studied.

\subsection{Heat transfer coefficient models}

We first investigated the use of the heat transfer coefficient model based upon the algebraic correlations from the literature, as described in the beginning of Section 3.1. While the Modelica tool was able to compile these models, it was unable to initialize or run simulations of these models due to failure of convergence for the nonlinear equation solver. While detailed information about the flattened model from dsmodel. mof is unavailable due to the use of encrypted refrigerant property models, statistics from the compilation process provide some indication of the cause of this failure: this information indicates that 37 numerical Jacobians are generated, which corresponds to the number of refrigerant volumes included in the cycle (27 volumes for the condensing heat exchanger and 10 volumes for the evaporating heat exchanger, corresponding to the number of tubes in each). As suggested in Section 3, the algebraic equations that couple the heat transfer coefficient to the pressure, specific enthalpy, and mass flow rate in each volumes are quite nonlinear and difficult to solve. While it may be possible to customize this correlation or otherwise provide sufficient information to the compiler (e.g., analytical derivatives via annotations or external functions) for the nonlinear solver to function correctly, the extra effort to do so for a specific set of correlations could be substantial, and would have to be replicated for every new correlation.

As the cycle models incorporating the correlation-based HTC models did not produce any viable simulations, we 
turned our attention to the use of the simplified HTC models. While the reduced nonlinearity of these closure models enabled the successful simulation of the cycle behavior, oscillations in the cycle behavior as seen in Figure 1 can occur due to either an input forcing function or simply the selection of an unlucky operating point. These oscillations are caused by the high gain of $d \alpha / d x$ in the transition region; small changes in the state variables are coupled to large changes in the heat transfer coefficient, which in turn has a measurable effect on the mass flow rate, pressures, and specific enthalpies. In this situation, these interactions can act to drive the system towards an unstable steadystate manifold and result in limit cycle behavior.

This behavior can be seen in Figures 5 and 6, which illustrate the flow quality and local refrigerant-side heat transfer coefficient in volumes 6 and 7 in the condenser for both the simplified algebraic HTC and the simplified dynamic HTC. For this heat exchanger, the HTC model is configured to have a liquid heat transfer coefficient of 378 $\mathrm{W} / \mathrm{m}^{2} \mathrm{~K}$ below a flow quality of 0 , a two-phase heat transfer coefficient of $4600 \mathrm{~W} / \mathrm{m}^{2} \mathrm{~K}$ between a flow quality of 0.1 and 0.9 , and a vapor heat transfer coefficient of 369 $\mathrm{W} / \mathrm{m}^{2} \mathrm{~K}$ above a flow quality of 1 , all at a mass flow rate of $36 \mathrm{~g} / \mathrm{s}$. It is evident that the algebraic HTCs in Figure 6 change abruptly when the flow quality illustrated in Figure 5 for either volume moves into the transition region. More specifically, the large spikes in the heat transfer coefficient for volume 6 are directly associated with the excursions in the transition region. These large gradients in the heat transfer coefficient are directly coupled to many other aspects of the flow. As the heat transfer coefficients change rapidly, the flow itself changes in such a way to reduce the flow quality and form this periodic behavior.

These oscillations in the heat transfer coefficient and flow quality can affect the overall cycle simulation in a variety of ways. Aside from the corresponding oscillations in other variables that are coupled to the heat transfer coefficient, these large gradients will also prevent the integration routines from taking large time steps, resulting in long simulation times. These effects can be mitigated somewhat for these simplified algebraic closure models in many circumstances by increasing the width of the transition regions, but the resulting gradual changes in the heat transfer coefficients can pose problems when linearizing models for control design (due to the high gains from the derivatives of the heat transfer coefficients) and also may not be justified by experimental data.

In this context of challenges posed by these algebraic heat transfer coefficients, we evaluated the performance of the vapor compression cycle with the dynamic heat transfer coefficient models. This study was performed by directly replacing the heat transfer model in the previous set of cycle models using the redeclare keyword, thus enabling a direct comparison of the performance of the method. The time constant $\tau$ of the dynamic heat transfer coefficient models was set to 3 seconds, though other values were also used successfully; in general, this time

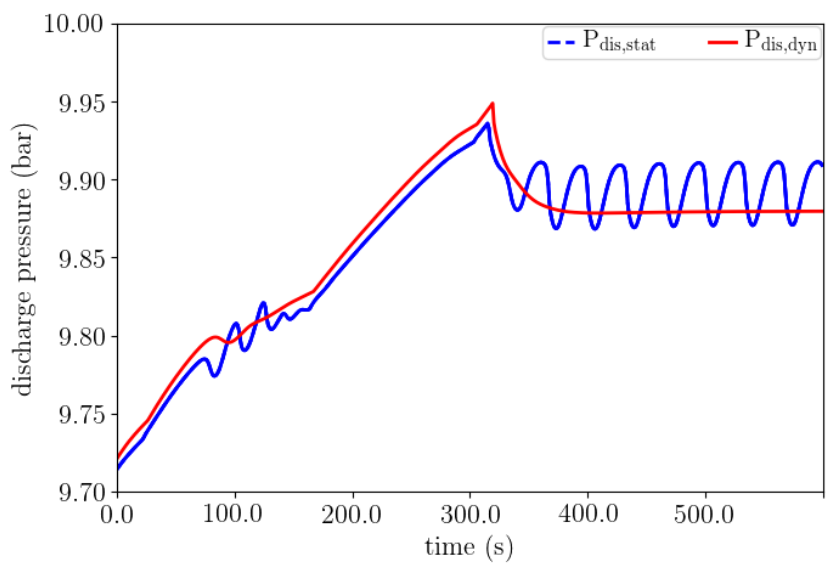

Figure 7. Compressor discharge pressure using simple heat transfer coefficient model with and without low-pass dynamics.

constant should be set to a relatively small value to minimize its affect on other cycle dynamics.

These new cycle simulations did not exhibit any oscillations, as can be seen in Figures 5 and 6, and also did not have any of the numerical Jacobians seen in the first set of algebraic heat transfer coefficient models. The lowpass behavior of the heat transfer coefficient has the effect of damping out these oscillations, allowing the system to converge to a steady-state operating point. Comparing both the flow quality and the local heat transfer coefficients for both volumes 6 and 7, the filtered value of these variables can be seen to be within the range of values for the previous simulations, suggesting that the cycle behavior with the dynamic closure model is not affected significantly. Moreover, Figure 7 illustrates behavior of the compressor discharge pressure both with and without the dynamic HTC model, confirming both that the oscillations in this pressure signal were caused by the heat transfer coefficient and that the dynamic HTC can successfully eliminate these oscillations. The addition of these dynamics had a minimal effect on the overall cycle simulations; the RMSE between the cycles with and without the dynamic HTCs was only $2.7 \mathrm{~W}$ out of a total cooling capacity of $1190 \mathrm{~W}$.

Table 3 illustrates the significant benefits that these dynamic HTC models can have on the cycle simulation time. While the correlation-based algebraic models were unable to simulate, a 1000 second simulation of the cycle with simplified algebraic models took 228 seconds to run due to the oscillations caused by the heat transfer coefficient model. Use of the dynamic HTC models reduced this computational time significantly; the dynamic version of the correlation model could be used and had a CPU time of 146 seconds, while the dynamic version of the simplified HTC model was able to simulate the entire $1000 \mathrm{sec}-$ ond duration in 39 seconds, representing an $83 \%$ reduction in CPU time. These results suggest that multiple HTC models could be used profitably in simulating the cycle; the simplified models could be used first to quickly study large-scale dynamics, and the more detailed correlations 


\begin{tabular}{lc} 
HTC Formulation & CPU Time (s) \\
\hline Algebraic, correlation-based & - \\
Algebraic, simplified & 228 \\
Dynamic, correlation-based & 146 \\
Dynamic, simplified & 39
\end{tabular}

Table 3. CPU time for alternate HTC formulations.

could then be used to obtain more accurate results.

\subsection{Pressure loss models}

The models of the pressure drop were tested in an analogous manner to those of the heat transfer coefficient. The base vapor-compression cycle model was used with the simplified dynamic heat transfer coefficient model, and the performance of the system with the friction-only, $d p / d t$, and linear pressure drop momentum balances was evaluated with both algebraic and dynamic closure models. Although the $d p / d t$ and linear pressure drop models neglect the frictional pressure loss term, the method described in Section 3 was used to decouple the mass flow rate from the pressure drop in each volume.

In general, frictional pressure drop correlations are written as $d p=f(\dot{M})$, so that the mass flow rate must be known to calculate the effective pressure drop. In correlations where $d p$ is a function of both $\dot{M}$ and other thermodynamic variables (e.g., $d p=f(\operatorname{Re}, \sigma, \ldots))$, these correlations will result in a set of nonlinear equations, and the addition of dynamics into the frictional pressure drop model will improve their performance in much the same way that the heat transfer coefficient models were improved. In these cases, we have found that the value of the time constant $\tau$ must be relatively small to avoid influencing the system dynamics because the mass flow rate exhibits relatively high frequency behavior with respect to the fastest dynamics of the cycle. Unfortunately, the dynamics of the modified system approach those of the unmodified system as $\tau$ is reduced, so that the simulation speed of a cycle model with very fast dynamic closure models will be nearly identical to the simulation speed of a cycle model with algebraic closure models.

Unlike the heat transfer coefficient models, there are many frictional pressure drop correlations available in which this relationship can be analytically inverted, so that $\dot{M}=f(d p)$. In these correlations, the mass flow rate can be directly calculated from the frictional pressure drop, and there is no need to solve a nonlinear set of equations. This is the case for many of the correlations commonly used to describe frictional pressure drop, and is also the case for the correlations used in this paper. As a result, the addition of dynamics to the frictional pressure drop models does not improve the performance for many standard formulations of the momentum balance, including the friction-only momentum balance used in this work.

In contrast to the friction-only momentum balance, the $d p / d t$ and linear pressure drop models described in Sec-

\begin{tabular}{lc} 
Model type & CPU time $(\mathrm{s})$ \\
\hline Standard & 2855 \\
Friction only & 111 \\
$d p / d t$ & 214 \\
Linear pressure & 175
\end{tabular}

Table 4. CPU time for variants of momentum balance with dynamic frictional pressure drops.

tion 2 have a tight algebraic coupling between the pressure drop between consecutive volumes and the mass flow rate through these volumes. A set of nonlinear algebraic equations that couple the pressures, specific enthalpies, and mass flow rates thus result, with the rather predictable consequence (at this point) that the system becomes very difficult to solve. Experiments with these momentum balances resulted in the same behavior observed with the correlation-based algebraic heat transfer coefficient models, in which there were 37 numerical Jacobians and the Modelica tool was unable to simulate the model. With the addition of dynamics to the pressure drop terms, the cycle behavior could be simulated in the same conditions used to evaluate the heat transfer coefficient models; Table 4 shows that the $d p / d t$ and linear pressure drop approximations of the momentum balance are significantly faster than the transient momentum balance, though they are roughly comparable to the friction-only momentum balance.

\section{Concluding Remarks}

In this work, we explored some of the computational considerations that relate to the implementation of heat transfer coefficient and frictional pressure drop models that are included in vapor-compression cycle simulations, and described three different modeling approaches that can potentially improve the speed of the overall cycle simulations at a low cost to physical accuracy. On the basis of our experience, we would recommend that heat transfer coefficient models include some simple dynamics, as this enables the direct use of correlation-based models, and the reduction in computational time is significant. In comparison, these methods do not improve the frictional pressure drop models dramatically except when simplified momentum balances such as the $d p / d t$ or linear pressure drop methods are used.

There are a variety of directions in which this work could continue. These methods could conceivably be applied to off-cycle simulation, as the small mass flow rates and pressure variations tend to cause high-frequency oscillations in these cases that are difficult to simulate. In addition, it would also be valuable to consider the experimental characterization of the dynamics of heat transfer, rather than solely characterizing the steady-state behavior. This could potentially be correlated with other relevant phenomena for practical vapor-compression cycles, such as the effect of the oil circulating with the refrigerant. 


\section{References}

ASHRAE. HVAC Systems and Equipment Handbook. ASHRAE, Atlanta, GA, 2008.

J.J. Brasz and K. Koenig. Numerical methods for the transient behavior of two-phase flow heat transfer in evaporators and condensers. Numerical Properties and Methodologies in Heat Transfer, pages 461-476, 1983.

Dassault Systemes, AB. Dymola 2018 FD01, 2018.

F.W. Dittus and L.M.K. Boelter. Heat transfer in automobile radiators of the tubular type. University of California Publications in Engineering, 2(13):443-461, 1930.

M.K. Dobson and J.C. Chato. Condensation in smooth horizaontal tubes. Journal of Heat Transfer, 120:193-213, Feb 1998.

H. Elmqvist, H. Tummescheit, and M. Otter. Object-oriented modeling of thermo-fluid systems. In 3rd International Modelica Conference. Linkoping, Sweden, 2003.

K.E. Gungor and R.H.S. Winterton. Simplified general correlation for saturated flow boiling and comparisons of correlations with data. Chem. Eng. Res. Des., 65:148-156, 1987.

J.M. Jensen. Dynamic modeling of thermo-fluid systems with focus on evaporators for refrigeration. $\mathrm{PhD}$ thesis, Technical University of Denmark, Department of Mechanical Engineering, 2003.

D.S. Jung and R. Radermacher. Prediction of pressure drop during horizontal annular flow boiling of pure and mixed refrigerants. International Journal of Heat and Mass Transfer, 32 (12):2435-2446, 1989.

C. Laughman, H. Qiao, and D. Nikovski. Kernel regression for the approximation of heat transfer coefficients. In Gustav Lorentzen Natural Working Fluids Conference, 2016.

C. Laughman, H. Qiao, S.A. Bortoff, and D.J. Burns. Simulation and optimization of integrated air-conditioning and ventilation systems. In Proceedings of the 15th IBPSA Conference, pages 1824-1833, 2017.

S. Levy. Two-phase flow in complex systems. New York: John Wiley \& Sons, 1999.

P. Li, H. Qiao, Y. Li, J.E. Seem, J. Winkler, and X. Li. Recent advances in dynamic modeling of HVAC equipment. Part 1: Equipment modeling. HVAC\&R Research, 20(1):136-149, 2014.

R.W. Lockhart and R.C. Martinelli. Proposed correlation of data for isothermal two-phase, two-component flow in pipes. Chemical Engineering Progress Symposium Series, 45:3948, 1949.

Modelica Association. Modelica specification, Version 3.4, 2017. URL www. modelica.org.

Modelon AB. Vapor Cycle Library User Guide, 2018. v2.1.

H. Qiao and C. Laughman. Comparison of approximate momentum equations in dynamic models of vapor compression systems. In Proceedings of the 16th International Heat Transfer Conference, 2018.
H. Qiao, V. Aute, and R. Radermacher. Transient modeling of a flash tank vapor injection heat pump system - part I: Model development. Int. J. Refrigeration, 49:169-182, 2015.

C.C. Richter. Proposal of new object-oriented equation-based model libraries for thermodynamic systems. $\mathrm{PhD}$ thesis, Technische Universität Braunschweig, Institut für Thermodynamik, 2008.

Peter Stephan, editor. VDI Heat Atlas. Springer-Verlag, 2010. 